$57 \mid 2016$

Innovations pédagogiques dans l'enseignement des langues étrangères : perspective historique (XVle-XXle siècles)

\title{
Une troisième « voie » pour apprendre les langues : la Ianua Linguarum de W. Bathe (1611)
}

Javier Suso López

\section{OpenEdition \\ Journals}

Electronic version

URL: https://journals.openedition.org/dhfles/4395

DOI: $10.4000 /$ dhfles.4395

ISSN: 2221-4038

\section{Publisher}

Société Internationale pour l'Histoire du Français Langue Étrangère ou Seconde

\section{Printed version}

Date of publication: 1 December 2016

Number of pages: $97-116$

ISSN: $0992-7654$

\section{Electronic reference}

Javier Suso López, "Une troisième « voie » pour apprendre les langues : la lanua Linguarum de W. Bathe (1611)", Documents pour I'histoire du français langue étrangère ou seconde [Online], 57 | 2016, Online since 01 February 2018, connection on 28 March 2023. URL: http://journals.openedition.org/dhfles/ 4395 ; DOI: https://doi.org/10.4000/dhfles.4395

This text was automatically generated on 28 March 2023.

All rights reserved 


\title{
Une troisième « voie » pour apprendre les langues : la Ianua Linguarum de W. Bathe (1611)
}

\author{
Javier Suso López
}

\section{Introduction}

1 La Ianua Linguarum de W. Bathe (1611) a été eclipsée par la Ianua Linguarum reserata de Comenius (1631) : comme l'indique O'Mahony, « it is paradoxical that Comenius should inadvertently have contributed to the lack of awareness of Bathe's work today » (1981: 135). Paradoxe d'autant plus injuste que Comenius a repris de nombreuses réflexions du Proemium et des 10 chapitres introducteurs bilingues latin-espagnol qui précèdent la Ianua (1611 : 25-39, pour le texte en espagnol) et qui, malheureusement, n'ont pas été reproduits dans leur intégrité dans les adaptations postérieures à la première édition. Cette méconnaissance se poursuit de nos jours, malgré l'existence de plusieurs études, dont celles de Sean O'Mahony (1981), de Sean O'Mathúna (1986), de Jean Caravolas (1980, 1986, 1991, 2002), de Francisco Torres (2000), de Gonzalo Fernandes (2004). Nous estimons que $\mathrm{W}$. Bathe continue d'être méconnu dans la littérature en français concernant l'enseignement des langues (étant donné que la plupart des études antérieures sont en anglais, en espagnol, en portugais).

2 Nous avons complété ces sources avec d'autres ouvrages qui s'occupent de l'enseignement des langues aux XVI ${ }^{\mathrm{e}}$ et XVII ${ }^{\mathrm{e}}$ siècles, dont les références se trouvent à la fin de cet article. Il est essentiel de connaître la biographie de W. Bathe pour mieux comprendre ses choix : nous renvoyons pour cela à S. Mathúna (1986) et à J. Caravolas (2002). 


\section{Une nouvelle organisation du contenu lexical d'une langue}

3 La Ianua Linguarum propose une organisation et une présentation nouvelles des contenus d'apprentissage de la langue latine (et par contrecoup, de l'espagnol ou de toute autre langue), et définit un outil pour la première étape de cet enseignement, l'apprentissage des mots (vocablos). Apprentissage qui se complète dans des étapes postérieures : l'apprentissage des règles (concordancia), des " phrases » (phraséologie) et de l'élégance (le style, les procédés rhétoriques), dont la Ianua ne s'occupe point :

El cuerpo de qualquiera lengua consta de quatro miembros principales, de vocablos, concordancias, phrases, y elegancia : los vocablos da el Diccionario, las concordancias la Gramatica, las phrases los autores, la elegancia pintan los Rhetoricos con sus figuras: llamamos phrases las propiedades, o particulares modos de hablar de cada lengua. (Bathe, 1611:29-30)

Nous reconnaissons ici la division des études de la Novissima Linguarum Methodus de Comenius (2005 [1648]) en quatre paliers (Vestibulum, Ianua, Atrium et Thesaurum), qui retracent métaphoriquement le cheminement d'arrivée, d'ouverture de la porte, d'accès au palais puis de prise en main du trésor, pour lesquels il compose des outils spécifiques où le vocabulaire et la grammaire se complexifient progressivement (Caravolas $1994:$ 360-364).

5 En effet, les mots (dont le catalogue complet nous est fourni par le dictionnaire) doivent être rassemblés dans des phrases: le ciment qui les noue est l'affaire de la concordance (dont les règles nous sont fournies par la grammaire). Mais aussi, les mots sont souvent réunis sous des modalités particulières, qui échappent aux règles de la grammaire : c'est le cas des expressions idiomatiques et manières de dire propres à chaque langue (les phrases, dont s'occupera par la suite la phraséologie). Finalement, Bathe propose des constructions spéciales, dans le but de «cultiver» la langue et la rendre plus belle et élégante. L'apprentissage complet d'une langue consiste donc à maîtriser ces quatre composantes, dans cet ordre qui établit une progression sûre : les mots, la concordance (ou congruité), les " phrases ${ }^{1}$ et les figures de rhétorique.

6 La Ianua comporte ainsi douze «centuries» (soit douze cents phrases, toutes numérotées, p. 42-130), chacune d'elles correspondant à un sujet thématiquement défini (vice et vertu, justice et injustice, sagesse et folie...) en disposant les phrases latines dans les pages paires et les phrases correspondantes en espagnol dans les pages impaires. L'ouvrage se complète par une longue Introduction bilingue également (sous forme de dix chapitres, p. 25-39), où Bathe et ses collaborateurs expliquent la composition de l'ouvrage, les fondements méthodologiques (" de tres caminos», « del camino medio ») et l'ordre qu'il faut suivre dans ce petit ouvrage («obrezilla»). À la suite des douze centuries, se trouve une annexe contenant des termes "équivoques » (ambiguis, "qui possèdent plusieurs sens », par exemple, caritas : charité et cherté), regroupés également par sujets (charité, justice, Dieu...) et qui sont repris également dans des sentences en latin-espagnol (p. 132-139), et une liste des termes latins utilisés dans les douze centuries, avec la traduction espagnole correspondante, mis en ordre alphabétique et avec l'indication de la centurie où ils sont utilisés (p. 141-215).

7 Quelles sont ces sentences? Le terme espagnol "sentencia», outre l'acception de "décision, jugement ou arrêt prononcé par un juge », signifiait tout d'abord la réunion d'une série de mots ayant un sens et exprimant une idée ${ }^{2}$, puis un «dicho grave y 
sucinto que encierra doctrina o moralidad" - «un dicton, un proverbe ou une expression grave et courte qui renferme une certaine vérité ou moralité »- (Diccionario de la RAE), acception qu'on retrouve également en français ("pensée (surtout sur un point de morale) exprimée de manière dogmatique ».

8 Nous reprenons certaines sentences (tirées du début de la première centurie, consacrée à la morale : vice et vertu ») pour en donner une idée (en y ajoutant une traduction « littérale » en français) :

\begin{tabular}{|l|l|}
\hline A solo Dios criador honra y gloria & A Dieu seul notre créateur, hommage et gloire \\
\hline La dificultad del principio se ha de tolerar & La dificultad del principio se ha de tolerar \\
\hline El principio es la mitad del todo & Le début c'est la moitié du tout \\
\hline Fácil es añadir a lo inventado & Fácil es añadir a lo inventado \\
\hline Lo peor se nos pega más tenazmente & Les pires choses se collent à nous de façon obstinée \\
\hline $\begin{array}{l}\text { Compañero gracioso vale por coche (esto es } \\
\text { alivia el camino) }\end{array}$ & $\begin{array}{l}\text { Compagnon joyeux en voyage vaut une voiture (rend } \\
\text { la route plus légère) }\end{array}$ \\
\hline
\end{tabular}

9 La présentation de la langue sous forme sentences a pour but de garantir une compréhension correcte des mots (" haziéndose también en sentencias comprehension de todos los vocablos ", 1611:26). Face aux catalogues de sentences morales ou de proverbes qui existaient auparavant («ay muchos que juntaron sentencias morales, $\mathrm{y}$ procuraron imprimirlas, sin pretender otra cosa ninguna »), la Ianua offre plusieurs avantages : d'un côté, le choix des sentences, qui sont nullement accumulées sans avoir égard aux lecteurs, mais en étant utiles et agréables ; d'autre part, la sélection des mots.

\section{Mots familiers, rares, fondamentaux et dérivés}

10 Tel qu'ils le disent dans cette «declaración de las lenguas" (ou linguistic enlightenment selon la traduction de O'Mahony, 1981) des dix chapitres introducteurs, la première opération pour $\mathrm{W}$. Bathe et ses collaborateurs a consisté à séparer les mots familiers et les mots fondamentaux (1611: 31), en laissant de côté les mots rares et les mots dérivés (colligilibus). Ils constituent ainsi quatre catégories de mots: familiers, rares, fondamentaux et dérivés. Disons tout d'abord que le terme "(vocablo) fundamental " dans la Ianua ne possède pas le sens actuel (équivalent à essentiel, de base, constitutif...), adopté par les auteurs du Français élémentaire (Gougenheim et al., 1956; transformé en Français fondamental en 1964). « Fundamental » s'oppose à « collegible » (ou dérivé) dans la Ianua, et pourrait donc s'assimiler à "primitif». Ainsi, les termes dérivés n'ont pas été retenus, puisque la parenté qu'ils gardent avec le mot dont ils sont issus rend facile leur compréhension (1611: 30).

11 Comment déterminer les termes familiers? Bathe et ses collaborateurs expliquent que certains mots peuvent être d'usage courant («familiares») dans certains milieux (la médecine par exemple), et très rares dans un autre milieu, que certains termes ne sont ni familiers ni rares mais "moyens", ou bien ni fondamentaux ni dérivés mais 
"mixtes ", etc. La répartition des termes du dictionnaire dans chaque catégorie doit prendre en compte sept critères, que regroupent les aspects sociaux et les aspects d'usage individuel d'une langue: le temps, le lieu, l'état (social standing, dans la traduction de O'Mahony, 1981 : 158), la signification (context), le style (register), le mode (l'écrit, l'oral) et l'opinion (1611: 32). Mais d'autres difficultés subsistaient: certains mots dérivés sont fondamentaux ; il y a une différence entre "familiar» et « usado » : ce n'est pas parce qu'un mot est usité (chez un auteur, par exemple) qu'il est familier ou courant. Ils proposent ainsi une définition claire et nette de ce qui est pour eux un terme familier (ou courant) : « por familiares y raros entendemos los mas frequentes, y menos frequentes » $(1611: 30)$ et " para que un vocablo sea familiar requierese mas que la cosa significada sea tal que cada día, o comunmente se offrezca hablar della entre aquellos, cuyo estado [social standing] mira el que haze la distincción de los vocablos » (1611 : 33). L'expérience de W. Bathe dans la pratique de différentes langues, dans des contextes d'usage différents, le mène à constater le degré de familiarité d'un mot dépend de sa fréquence d'usage à l'intérieur d'un contexte (" estado ») particulier, et change selon les lieux et le temps... Et c'est ainsi qu'il entreprend de restreindre les limites pour procéder à la sélection du vocabulaire, en choisissant le contexte d'usage du latin à Louvain parmi les étudiants à qui il enseignait le latin (qui parlaient le latin comme s'il s'agissait de leur langue maternelle: «usan en la platica familiar de la lengua Latina de mejor gana que de la materna») qu'il prend comme base de sa sélection de mots fréquents (1611: 32). Aussi, comme O'Mahony l'indique :

he obviously identified what in our own day has come to be known as available vocabulary or mots disponibles. These words, though not necessary frequent, readily come to mind whenever there is occasion to discuss the concepts with which they are clearly associated. $(1981: 135)$

12 La prudence guide ainsi ses réflexions : il ne propose aucune formule scientifique en tant que savant, et se limite à faire une proposition en la matière ${ }^{3}$. Il divise ainsi le dictionnaire (il utilise le terme Calepin par antonomase) en termes familiers et rares, puis il ne retient que les mots familiers fondamentaux (1611:30). Aussi, il élimine les mots qui se rapportent à des vilaines choses («cosas feas»), qu'on ne peut pas nommer selon les règles de la bienséance, même s'ils sont très usités (1611: 34$)$.

Comme résultat de ce travail, 4827 mots différents (selon Fernandes, 2004:173; 5200 pour O'Mahony, 1981 : 133) sont sélectionnés. W. Bathe et ses collaborateurs ont ainsi entrepris une deuxième proposition, dans l'histoire d'une linguistique appliquée avant la lettre, du vocabulaire fondamental d'une langue, de la langue latine, en principe, mais qui s'étend à n'importe quelle autre langue par le seul fait de la traduction correspondante. Rappelons que la première proposition, dans le domaine hispanofrançais, est celle de Liaño (ou Ledel) qui propose dans son Vocabulario de 1565 un total de 1450 termes. Tout le chapitre quatre s'étend sur cette question essentielle, qui est au cœur de leur « artificio de palabras » (1611: 128). La limitation des termes (vocablos) qui sont incorporés aux sentences permet en effet pour eux un apprentissage rapide et effectif de la langue, c'est-à-dire de la comprendre et de la parler.

Une étude détaillée et comparative de ces deux entreprises lexico-didactiques serait certes intéressante, mais exigerait la composition d'un autre article. Nous nous limitons ici à avancer certains points. Ainsi, à titre d'exemple, dans le lexique de W. Bathe, parmi les 454 mots qui commencent par la lettre A en latin, quelque 265 sont des substantifs (58\%), 35 sont des adjectifs (7,7\%), 123 des verbes (27\%) et 32 des mots invariables - adverbes, prépositions, conjonctions, interjections - (7\%). À titre de 
comparaison, dans le lexique de Liaño/Ledel (1450 mots), parmi les 104 mots commençant par la lettre A, 30 sont des substantifs (30\%), 8 sont des adjectifs (8\%), 43 sont des verbes (42\%) et 23 des mots invariables et expressions (23\%). Disons également que le Français fondamental (1964), composé de quelque 1500 mots, comporte à la lettre A 87 mots, dont 33 substantifs (38\%), 4 adjectifs (4,6\%), 24 formes verbales (27,6\%) et 22 mots invariables $(25,2 \%)$. Nous voyons ainsi que chez Bathe les mots considérés fréquents sont trois fois plus nombreux, et que les substantifs - qui désignent des objets - sont surreprésentés par rapport au Vocabulario de Liaño et au Français fondamental. D'autre part, si les mots dérivés sont éliminés (du type turbar, perturbar, conturbar, desturbar, turbado, turbardor, turbación...), on ne voit pas pourquoi il retient le doublon amo (aimer) et amor (l'amour), appeto (désirer) et appetitus (le désir), arbitror (juger) et arbiter (le juge) ou ambigo (douter) et ambiguus (douteux)...

Bathe et ses collaborateurs entreprennent ainsi une toute première tentative d'analyse objective du lexique d'une langue ou mieux, de la langue, de toute langue. La conception de la langue qui préside la Ianua est celle de l'époque: la langue est l'instrument par lequel les choses qui existent sont désignées (signum) et donc chaque objet doit se correspondre à un terme (vocablo), et à l'inverse, conception qui repose sur considération de la langue comme une nomenclature de la réalité (ou isomorphisme ontologico-linguistique, cf. Luque Duran, 2004: 358-360). Le mouvement de la grammaire spéculative du XIII ${ }^{\mathrm{e}}$ siècle avait rapproché les grammaires de Donat et de Priscien de la logique aristotélicienne : rappelons que pour Aristote, les concepts (ou images mentales) sont tous les mêmes chez les hommes; et les mots ne sont que les symboles ou signes de ces «impressions " (images, représentations) de l'âme. Au-delà des différences dans les termes (vocablos), propres à chaque langue, il est possible de retrouver des identités dans les concepts, puisque ceux-ci sont les mêmes pour tous les hommes, comme nous l'avons dit. Avec la redécouverte du grec et de l'hébreu, les discussions au $\mathrm{XVI}^{\mathrm{e}}$ siècle prennent comme objet dans toute l'Europe quelle est la première langue et quelle est la langue parfaite (c'est-à-dire, quelle est langue qui représente le mieux la réalité, $c f$. Eco, 2004). Les grammairiens espagnols de l'époque Huarte de San Juan, Sebastián de Covarrubias, Francisco Sánchez, el Brocense (auteur de la Minerva, sive de causis lingua latinae, 1587) - défendaient d'autre part une interprétation littérale de la Genèse, le don d'une langue par Dieu aux hommes (en principe, l'hébreu), et donc aussi le rapport consubstantiel du mot à la chose (qui révélerait son essence), perdu par le foisonnement des langues (Babel). Les langues, au fil du temps, s'étaient éloignées de cette constitution primitive (de la part du premier architecte de la langue, Dieu lui-même), en spécial les langues vulgaires, qui sont pour Bathe moins «régulières" que les langues anciennes (1611: 38), et donc, peuvent mieux s'apprendre sans règles.

À partir de cette représentation mentale du rapport de la langue aux choses, W. Bathe et de ses collaborateurs entreprennent la tâche de déterminer la signification des mots qu'ils proposent dans leurs sentences, ce qui n'était pas une entreprise dénuée de controverses, puisque les mots tels qu'ils sont repris dans les dictionnaires (en tant que lemmes) signifient différemment en fonction des critères exposés (temps, lieu, contextes, registres...). Bathe prend des distances quant aux spéculations des grammairiens de leur époque, et ne remonte pas au prétendu sens étymologique (qui serait donc premier et fondamental); il se limite à indiquer que les mots ne signifient pas de manière unique, et qu'il faut restreindre la « latitude » des emplois en fuyant les extrêmes et en choisissant le sens moyen « al cual según el juyzio del prudente mas se 
acercan » (1611:32), pour employer ses propres mots du préambule (1611:32). Les limites géographiques (Louvain) et sociales (les étudiants de lettres d'humanités) garantissent selon lui, d'une part, les manières de parler courantes et, d'autre part, les mots « moyens » ou « mixtes ».

Une fois acquis les mots familiers et fondamentaux, comment s'y prendre pour les autres ? La question est posée dans le dernier chapitre du Préambule. La Ianua ne garantit l'apprentissage que de très peu de mots d'une langue (même pas cinq mille !). Le chapitre dix veut répondre à cette probable objection des détracteurs de la méthode, et au-delà de sa réponse - il est possible de continuer de faire des sentences semblables avec les mots rares, même si on répète les verbes, qui sont moins nombreux que les substantifs - c'est la conception de la langue traditionnelle qui y est reflétée, ainsi que la signification de son entreprise (artificio).

\section{La composition des sentences et leur ordonnement}

18 Aussi, si une langue se compose d'un ensemble de mots, savoir une langue consiste donc à connaître le vocabulaire de cette langue. Pour cela, le mieux c'est d'insérer les mots dans des sentences. En effet, les termes sélectionnés ne sont pas disposés dans les sentences et les centuries n'importe comment; il fallait le faire de façon telle qu'ils soient « entendidos clara, y distinctamente " $(1611: 32)$. Les sentences devaient ainsi être très brèves ; mais aussi, aucun des termes ne devait être répété (sauf bien sûr les mots grammaticaux) et il fallait que les termes y soient rassemblés dans un ordre fort bien étudié.

Les sentences sont ainsi distribuées thématiquement selon l'ordre des principes moraux (les différentes vertus et leurs antithèses), en comportant (selon l'intention de Bathe) une maxime morale substantielle, rapportée à des questions agréables (« en las primeras se esparcen a cada paso muchas cosas agradables por curiosidad»), et sous une forme pleine de "donayre y gracia " $(1611: 34)$. Bathe se rend bien compte que toutes les sentences ne remplissent pas au même degré ces conditions : c'est le cas de la neuvième centurie (intitulée précisément: "des objets qui appartiennent à cet artifice ", De artificialibus), de la dixième et de la onzième centuries (intitulées toutes deux « des divers objets ", De indistinctis), où sont reprises pêle-mêle les différentes professions et les outils propres à chacune d'elles, sous forme de phrases absolument artificielles. Ainsi : «Les manches liées avec une ficelle, la bonne sépare avec un tamis la fleur de la farine du son» (sentence 852)... Des sentences morales, des vérités générales et des descriptions archétypiques y apparaissent également telles que : «le crottin des pigeons donne vie aux vergers » (sentence 734), « le sommeil est une image de la mort » (751), «la femme qui vit en concubinage doit être fouettée et battue » (1008)... De même, Bathe indique que, quand il a dû s'occuper des adverbes et autres mots indéclinables, la qualité de l' « artificio » s'en ressent, et les sentences changent de nature, en faveur de petites phrases (oraciones), qui sont enchaînées les unes les autres, ou bien en faveur de longues phrases composées de plusieurs parties. Finalement, la dernière centurie reprend la liste des sentences déjà traduites en espagnol sous forme de "discours rhétorique» (traducción paraphrastica) sous le titre de "Contre Zoïle, l'envieux ou le détracteur " (censeur d'Homère), où les phrases auparavant éparses sont réunies sous forme d'un texte cohérent. La présentation des phrases sous forme de discours se retrouve au milieu de la cinquième centurie, dans le « Discours de la passion 
de J.- Christ » (sentences 455-500). La composition se complète, comme nous l'avons indiqué, par une liste des mots familiers choisis disposés en ordre alphabétique (latinespagnol), avec indication du numéro de la sentence où ils sont utilisés, et une annexe contenant des termes "équivoques ", comme nous l'avons déjà indiqué.

Dans la mesure où les phrases (sentencias) regroupent les termes d'une façon ordonnée et systématisée, leur mémorisation est donc rendue plus facile et plus effective. Ce chemin nouveau (via tertia, 1631: 33) possède donc les avantages de proposer des manières de parler faciles puisqu'elles sont courantes (comunes) et vraies ou correctes (ciertas). Nous pourrions discuter, certes, sur les critères qui ont mené Bathe et ses collaborateurs à adopter l'ordre dans lequel les centuries sont établies (et donc, l'ensemble du vocabulaire). Pour eux, cette disposition reflète l'architecture de la nature, qui est appréhendée et classée de manière rationnelle. En tout cas, une logique de classement est proposée, face à la disposition alphabétique des dictionnaires de l'époque, ou la disposition des recueils lexicaux thématiques de l'époque.

21 Nous trouvons ici un principe essentiel de la démarche de Bathe, inspiré de Platon luimême, et qui sera repris par Descartes dans son Discours sur la méthode : « ordo est anima rerum » : l'ordre est le principe vital des choses, l'âme des choses, la marque de l'esprit qui les atteint (« el orden es animo de las cosas », dans la traduction espagnole, 1611 : 29). Avec le corollaire suivant: apprendre les choses dans un ordre logique garantit l'efficacité de la méthode. En effet, cet ordre repose sur un apothtegme méthodologique rationnel : l'« empire de la raison» (el imperio de la racionalidad) qui nous permet de reconnaître dans la langue un ordonnement intrinsèque, qui exige que «nous devons en premier lieu prendre en compte la finalité que nous nous proposons, en second lieu les moyens, ensuite, la mise en œuvre (ou l'exécution), et finalement les circonstances de cette mise en œuvre » $(1611: 29)^{4}$.

Tout le discours préliminaire de la Ianua est traversé par la rationalité de leur démarche: partout dans le monde, et partout dans une langue, on trouve l'ordre admirable de l'architecture de son créateur immortel ( $«$ la architectura de aquel immortal artifice", ibid.). L'auteur de la Ianua et ses collaborateurs ne font que découvrir et mettre en lumière cet ordre à travers leur artificio. Le critère qu'ils adoptent pour le classement des mots en centuries et l'ordre des centuries lui-même part du Créateur, à partir des quatre vertus cardinales, et ensuite d'un classement des actions humaines, et finalement des êtres et objets de la nature: vertu et vice; prudence et imprudence; tempérance et intempérance ; justice et injustice ; fortitude et faiblesse; des actions humaines; des choses qu'on fait avec impétuosité ou tranquillité; des êtres vivants et des choses non vivantes; des choses propres à cet artifice ; objets divers ; Contre Zoïle... La portée morale est ainsi une part constitutive de l'apprentissage langagier.

\section{L'ordre de la langue}

23 Ainsi, le corps (cuerpo) de n'importe quelle langue est composé de quatre membres (miembros) : les mots, la concordance, les " phrases » et l'élégance, comme nous l'avons indiqué auparavant. Cette distribution contredit la disposition des ars grammaticaux de l'époque: lettres, analogie (mots: morphologie), syntaxe (construction et concordance), prosodie et style. Bathe n'est pas un grammairien, mais un pédagogue, 
qui se rend compte que l'apprentissage d'une langue selon l'ordre canonique de la grammaire ne menait nullement à la maîtrise effective de la langue (la parler).

Bathe revendique que sa méthode traite les mots de la langue de la même façon où la grammaire traite les règles : en les mettant en ordre, en suivant un chemin régulier, ce qu'aucun auteur n'avait jamais proposé auparavant. Cet ordre est complètement différent de celui proposé par les dictionnaires à l'usage au XVI ${ }^{\mathrm{e}}$ siècle, qui suivaient un ordre alphabétique comme nous avons indiqué auparavant. Pour Bathe, l'essentiel est de connaitre les mots "fundamentales »; ensuite, il est facile d'apprendre le sens des mots dérivés. Face au chemin irrégulier, où la rencontre d'un nouveau mot, " fundamental », dépend des caprices du hasard (des conversations, des textes : « oyendo, hablando o leyendo »), la Ianua offre la garantie au débutant (el bisoño o novicio) de trouver réunis tous les mots dont il aura besoin, dans cette espèce d'arche de Noé des mots que constitue la Ianua (métaphore qui est utilisée par Bathe, 1611 : 27).

\section{L'apprentissage régulier du vocabulaire}

Ces limites n'empêchent point la validité de la Ianua : la mémorisation des mots tels qu'ils étaient disposés dans les recueils lexicaux de l'époque (Vocabularios), sous forme de listes alphabétiques ou thématiques, était une entreprise très coûteuse, en termes de temps, et peu efficace, en termes de rendement, puisque les mots ne possèdent pas de "sens ", et la mémoire, ne possédant aucune aide pour leur compréhension, ne peut point les retenir ${ }^{5}$. Le « sens » est donc l'affaire non pas du mot isolé, mais de la phrase, qui reflète une idée complète: nous retrouvons ici une idée chère à la tradition linguistique de l'Espagne, que la Grammaire générale et raisonnée de Port-Royal (1660) fera sienne également. Comenius reprendra cette idée en employant la métaphore suivante : si vous construisez un mur en y ajoutant des pierres l'une au-dessus de l'autre, ce mur s'écroulera certainement ; pour qu'il tienne, il faut mettre de la chaux pour faire tenir les pierres entre elles (1631: Préface); les phrases nouent ainsi les mots tel que le ciment noue les pierres. C'est sûr que la méthode prônée par la Ianua exige une mémorisation non moins forte; mais, du moins, à en juger par les témoignages recueillis au début de l'ouvrage (1611:1-4), les résultats étaient plus précoces et plus voyants. En bref, la « via media » de W. Bathe consiste à apprendre les mots dans un ordre, insérés dans des phrases.

\section{La congruité, les « phrases » et l'élégance}

La Ianua ne prétend pas donc faire acquérir aux apprenants de la grammaire, des "phrases» ou l'élégance de la langue, étapes nécessaires mais postérieures dans l'apprentissage de toute langue; cependant, selon l'auteur de la Ianua et ses collaborateurs, l'apprentissage des mots à travers les sentences va préparer de manière effective ces autres apprentissages. Dans le chapitre six du préambule (De la congruité), ils soutiennent que les règles de grammaire peuvent être apprises sous forme de sentences, d'une manière plus facile qu'à travers les règles dénudées de la grammaire ${ }^{6}$. Et ceci est valable surtout pour l'apprentissage des langues vulgaires, dans lesquelles (nous traduisons) «il n'est pas si nécessaire d'apprendre de manière scientifique les choses qui ont rapport à la méthode de la grammaire » (1611: 35$)$, où il suffit de parler avec correction (congruidad) même si on ne connaît pas la règle qui y est appliquée. 
Tandis que pour les langues latine, grecque et hébraïque, cette voie moyenne ne sera pas suffisante, et il faudra connaitre les préceptes de façon scientifique. Bathe annonce un deuxième ouvrage où ils montreront comment rassembler les sentences pour en extraire tout ce qui appartient à la finalité de parler avec congruité, ouvrage qui n'a pas été mené à bout. L'idée est cependant que les règles de grammaire sont contenues implicitement dans les sentences proposées, et que l'apprentissage des sentences mène à une maîtrise de la langue quant à sa correction.

De même, dans le chapitre sept (De las phrases, y elegancia, 1611 : 36), Bathe annonce un ouvrage spécifiquement consacré à l'apprentissage des "phrases » et de l'élégance. La vision mécanique de la langue se poursuit jusqu'au but: de la même façon que les lettres se combinent pour former des mots et que les mots se combinent pour former des sentences, les sentences se combinent pour former des «phrases» et des discours suivis (l'élégance). L'exécution de cet ouvrage exige un grand travail et la collaboration d'autres «ouvriers» («la execucion requiere muchos obreros para hazerse bien»); il montre toutefois à deux reprises des exemples de cette réalisation (le Discours du Christ et le Contre Zoile), tout en renvoyant aux ouvrages de Paulo Manucio (éditeur, imprimeur et humaniste italien du XVIe siècle). Le procédé se compose de deux phases : la réunion de phrases d'auteurs sur un même sujet; puis l'assemblage des phrases en évitant la répétition des mots.

\section{En guise de conclusion}

D'autres questions ont été laissées de côté dans cette analyse, que nous avons juste effleurées, auxquelles nous reviendrons prochainement. Nous nous sommes limités à exposer la partie du quoi : quoi enseigner, dans quel ordre ? Il faudrait compléter cette analyse avec le comment: quelles actions doivent-ils entreprendre, autant le maître que le disciple? Aussi, les sentences de la Ianua Linguarum sont porteuses d'un contenu culturel et moral, auquel les buts de l'éducation linguistique contribuent de façon essentielle. Ou encore, l'entreprise (artificio) de W. Bathe dans la Ianua dépasse la simple idée de constituer un lexique fondamental (à travers la liste des mots courants) et leur insertion en sentences irréprochables, pourvoyeuses de la vérité : les douze cent sentences constituent également un catalogue des idées claires et justes qu'une personne peut concevoir dans son esprit pour la représentation du monde. Ce double catalogue (de mots et d'idées) est à mettre en rapport avec les tentatives pour fuir de la malédiction de Babel et créer une langue parfaite (permettant de convertir les infidèles) ${ }^{7}$. Le catalogue de sentences, et donc d'idées, annonce la prétention de faire correspondre de façon fixe la forme du contenu à la substance du contenu, et donc, d'ordonner le contenu à penser et à dire, ce qui est fait non par une langue nouvellement recréée, mais en latin (vouée par les Jésuites à récupérer le statut de langue de la communication universelle) et, de surplus, dans les autres langues, à travers la traduction littérale des sentences. L'élimination des modifications que subit la substance du contenu dans le passage à l'expression dans les différentes langues (donc, dans la forme du contenu) serait le résultat final de cette entreprise et, donc, le rapprochement de la langue primitive.

Bien sûr, rien de tout cela n'est explicité. Les Jésuites ne voulaient absolument pas avoir de conflits avec l'orthodoxie, et n'importe quelle proposition nouvelle pouvait être vue comme hétérodoxe de la part des philosophes-théologues traditionalistes, très 
puissants à Salamanque: l'auteur de Minerva lui-même, Sánchez de las Brozas, professeur de rhétorique à Salamanque, avait été mené par trois fois à un tribunal de l'Inquisition (en 1584: il avait "critiqué » la forme littéraire des évangiles ! Puis en 1595 et en 1600) ; ce dernier jugement est interrompu par sa mort alors qu'il avait 78 ans. C'est pourquoi, peut-être, que les Jésuites ne portèrent pas une grande attention à la Iana Linguarum de Bathe, et que Comenius, par contre, adopta avec enthousiasme sa proposition, tout en corrigeant certains aspects, qui ne modifient pas l'idée centrale.

\section{BIBLIOGRAPHY}

\section{Sources primaires}

BATHE, William (1611). Ianua linguarum, sive modus maxime accommodatus, quo patefitaditus ad omnes linguas intelligendas. Industria partrum hibernorum Societatis Iesu [...] in qua totius linguae vocabulae, quae frequentiora et fundamentalia sunt continentur: cum índice vocabularium et translatione Hispanica ejusdem tractatus. Salamanticae : apud Franciscum de Cea Tesa.

Principales adaptations :

-1615. Ianua Linguarum. Londres : William Welde (latin-anglais).

-1617. Ianua Linguarum Quadrilinguis, or a Messe of Tongues : Latine, English, French, and Spanish. Londres : Jean Barbier.

-1629. Ianua Linguarum Silinguis. Strasbourg : I. Habrecht.

-1631. Porta Linguarum trilinguis reserata et aperta. Londres : Georgius Millerisu (latin, anglais, français).

-1631. Ianua Linguarum Reserata et Aperta. Lezno, s.n., par Comenius (latin, français, allemand).

-1637. Mercurius Quadrilinguis. Padoue : par Gaspar Sciopius (latin, grec, hébreu, italien) et Bâle (l'allemand y substitue l'italien).

BELLOT, J. (1588). The French method [...]. Londres : Robert Robinson.

COMENIUS, Jan Amos (2005 [1648)]. Novissima Linguarum Methodus. La toute nouvelle méthode des langues. Genève-Paris : Droz.

LIAÑo, Iaques (Ledel) (1565). Vocabulario de los vocablos que mas comunmente se suelen usar... El estilo de escrivir, hablar y pronunciar las dos Lenguas, el Francés en Castellano y el Castellano en Francés. Alcalá de Henares : Francisco de Cormellas y Pedro de Robles.

VALDÉs, Juan de (1535). Diálogo de la lengua. Rééd. Buenos Aires : Ed. Sopena, 1940 ; José F.

Montesinos, Madrid : Espasa-Calpe, 1976 ; Madrid : Olympia ediciones, 1995.

Sources secondaires

AUROUX, Sylvain (1994). La révolution technologique de la grammatisation. Bruxelles : Mardaga.

BREVA-CLARAMONTE, Manuel (1994). La didáctica de las lenguas en el Renacimiento : Juan Luis Vives y

Pedro Simón Abril. Bilbao : Universidad de Deusto. 
CARAVOLAS, Jean (1994). La didactique des langues. Précis d'histoire I (1450-1700). Montréal-Tübingen : Presses de l'Université de Montréal-Gunter Narr Verlag.

-(1995). « Apprendre une langue étrangère à la Renaissance ». Historiographia linguistica, XXII, 3 , 275-307.

-(2002). « On Bathe's and Comenius' Ianua linguarum : The errors, the Myths and the facts ». Acta Comeniana, 15-16, 39-84

CORCUERA, J. Fidel \& GASPAR, Antonio (1999). La lengua francesa en España en el siglo XVI [...].

Zaragoza : PU.

DE CLERQ, Jan, LIOCE, Nico \& SWIGGERS, Pierre (éds.) (2000). Grammaire et enseignement du français 1500-1700. Louvain : Peeters.

ECO, Umberto (1994). La recherche de la langue parfaite dans la culture européenne. Paris : Seuil.

ESPARZA TORRES, Miguel Ángel. et al. (2008). Bibliografía temática de historiografía lingüística española : Fuentes secundarias, 2 vol. Hambourg : BuskeVerlag.

FERNANDES, Gonçalo (2004). « A Ianua Linguarum dos Jesuítas irlandeses (Salamanca, 1611) e a

Porta de línguas de Amaro de Roboredo (Lisboa, 1623) ». Boletim de Estudos Clássicos, 42, 165-180.

HOLTZ, Louis (1981). Donat et la tradition de l'enseignement grammatical, étude et édition critique. Paris :

CNRS.

HOWATT, Anthony Philip Reid (2004 [1984). A History of English Language Teaching. Oxford : OUP.

KIBBEE, Douglas A. (1989). « L'enseignement du français en Angleterre au XVI ${ }^{\mathrm{e}}$ siècle ». In P.

Swiggers et W. Van Hoecke. La langue française au XVI $I^{e}$ siècle : usage, enseignement et approches descriptives. Louvain : University Press, 54-77.

KOERNER, E.F.K, NIEDEREHE, Hans-Josef. \& QUILIS, Antonio (2001). History of linguistics in Spain : Historia de la lingüística en España (II). Amsterdam : John Benjamins.

KUKENHEIM, Louis ([1932] 1974). Contributions à l'histoire de la grammaire italienne, espagnole et française à l'époque de la Renaissance. Utrecht : H\&S Publishers.

LUQUE DURÁN, Juan de dios (2004). Aspectos universales y particulares del léxico de las lenguas del mundo. Granada : Impredisur.

NIEDEREHE, Hans-Josef (1995). Bibliografía cronológica de la lingüística, la gramática y la lexicografía del español (BICRES I) : desde los comienzos hasta el año 1600. Amsterdam, [etc.] : John Benjamins.

NIETO JIMÉNEZ, Lidio (1988). « Estudio introductorio », Reglas gramaticales para aprender la lengua española y francesa, de A. del Corro, de 1586. Madrid : Arco Libros.

O’MAHONY, Sean (1981). « The Preface to William Bathe's Lingua Linguarum (1611) ». Historiographia Linguistica, VIII : 1, 131-164.

O’MATHÚNA, Seán P. (1986). William Bathe, S.J., 1564-1614 : A pioneer in linguistics. (English translation from the Irish edition, Dublin, 1981). Amsterdam/Philadelphia : John Benjamins Publishing.

PEREA SILLER, Francisco Javier (1998). Fray Luis de León y la lengua perfecta. Códoba : Camino. SARMIENTO, Ramon (dir.) (1987). « La tradition espagnole d'analyse linguistique ». Histoire, Épistémologie, langage, t. 9, II.

SUSO LÓPEZ, Javier (2009). « Conception de la langue et l'enseignement/apprentissage des langues au XVI ${ }^{\mathrm{e}}$ siècle ». Le Langage et l'homme. Revue de didactique du français, XXXXIV.1, 111-122. 
SWIGGERS, Pierre (2006). « El foco 'belga': Las gramáticas españolas de Lovaina $(1555,1559)$ ». In J. Gómez Asencio (dir). El castellano y su codificación gramatical. Vol. I : De 1492 (A. de Nebrija) a 1611 (John Sanford), Burgos/Salamanca : Fundación Instituto Castellano y Leonés de la Lengua, 161-214. SWIGGERS, Pierre \& VANVOLSEM, Serge (1987), « Les premières grammaires vernaculaires de l'italien, de l'espagnol et du portugais ». Histoire, Épistémologie, langage, IX-1, 157-181.

\section{NOTES}

1. Le terme espagnol phrase (ainsi écrit à l'époque) désignait «les propriétés, ou les modes particuliers de parler de chaque langue » («propiedades, o particulares modos de hablar en cada lengua» (Bathe, 1611: 30), et avait donc un sens proche d'idiotisme. En français, le terme "phrase " possède également cette acception au XVI ${ }^{\mathrm{e}}$ siècle (tour, expression), et prend une acception particulière dans le domaine linguistique au XVII ${ }^{\mathrm{e}}$ siècle (en tant qu'ensemble de mots possédant un sens), acception qui sera empruntée par l'espagnol (frase), et rivalise avec le terme oración.

2. On trouve cette acception par exemple chez Nebrija : "Verbo es una de las diez partes de la oración, el cual se declina por modos et tiempos, sin casos. E llámase verbo, que en castellano quiere dezir palabra, no por que las otras partes de la oración no sean palabras, mas por que las otras sin ésta no hazen sentencia alguna, ésta, por ezcelencia » (1492 : chap. X.).

3. Ainsi, le texte reflète cette attitude intellectuelle à l'aide de conditionnels, de mots adversatifs et modulateurs (il faut remarquer, cependant, on pourrait, il convient... etc., voir principalement le chapitre quatre de l'Introduction, p. 32-33), et surtout dans la phrase : « Donc, pour procéder avec prudence dans cet ouvrage...».

4. "Que primero avemos de tener cuenta del fin, lo segundo de los medios, luego de las execuciones, que es poner por obra, y finalmente las circunstancias ».

5. « Las palabras puestas en el Vocabulario ningún sentido tienen : de donde nace que la memoria desamparada de layuda del entendimiento no los retiene » $(1611: 30)$.

6. «[las reglas] se pueden tambien enseñar comodamente en sentencias, de manera que mas facil, cierta, y prestamente se peguen en los animos, que se puede hazer por desnudas reglas de Gramatica solamente ».

7. Ces tentatives surgissent en Europe dès les XIII ${ }^{\mathrm{e}-X I V}{ }^{\mathrm{e}}$ siècles (l'Ars Magna, de Raymond Lulle, par exemple, qui est l'objet d'un regain de l'intérêt dans l'Espagne confrontée à la tâche de conversion des infidèles au XVI ${ }^{\mathrm{e}}$ siècle ; Philippe II appuiera sa béatification).

\section{ABSTRACTS}

This article claims to expose of what consists the average way of languages teaching proposed by the Ianua Linguarum de W. Bathe (1611). Bathe proposes a new learning language learning material with a new organization of the lexicon (classified in the form of familiar, rare, fundamental and derived words), which is introduced into orderly "judgments" thematically, not through "situational" criteria as in the colloquia, but according to the order of the moral virtues. The third "average way" is not thus an eclectic method, as a middle way between a regular method (by rules) and an irregular one (by the use), but a way where we learn the vocabulary in 
an orderly way (and also the correction of the language) and the "style") through the memorization of judgments. We so consider that Ianua de Bathe is a work which merits a large place in the theoretical reflection on the education / learning of the languages in the $17^{\text {th }}$ century.

Cet article prétend exposer en quoi consiste la «voie moyenne » d'enseignement des langues proposée par la Ianua Linguarum de W. Bathe (1611). Bathe propose un matériel d'enseignement de la langue nouveau (language learning material) avec une organisation nouvelle du lexique (classé sous forme de mots familiers, rares, fondamentaux et dérivés), qui est introduit dans des " sentences » ordonnées thématiquement, non pas à travers des critères « situationnels » comme dans les colloques, mais selon l'ordre des vertus morales. La troisième " voie moyenne » n'est donc pas une méthode éclectique, à mi-chemin entre méthode régulière (par les règles) et irrégulière (par l'usage), mais une voie où l'on apprend le vocabulaire de façon ordonnée (et aussi la correction de la langue et le "style ", mais de façon implicite) à travers la mémorisation de sentences. Nous considérons ainsi que la Ianua de Bathe est un jalon important dans la réflexion théorique sur l'enseignement/apprentissages des langues au XVII ${ }^{\mathrm{e}}$ siècle.

\section{INDEX}

Mots-clés: enseignement des langues, outil d'enseignement, matériel langagier, lexique, sentence, voie moyenne, $\mathrm{W}$. Bathe, XVIIe siècle

Keywords: language teaching, linguistic teaching materials, lexicon, phrase, judgment, average way, W. Bathe, 17 th century

\section{AUTHOR}

\section{JAVIER SUSO LÓPEZ}

Universidad de Granada

jsuso@ugr.es 